\title{
Giant frontal sinus osteoma and its potential consequences: illustrative case
}

\author{
Riyaq A. Farah, MBBS, ${ }^{1}$ Arturo Poletti, $\mathrm{MD},{ }^{2}$ Aaron Han, $\mathrm{MD}, \mathrm{PhD},{ }^{3}$ and Ramon Navarro, $\mathrm{MD}^{4}$ \\ ${ }^{1}$ College of Medicine, Gulf Medical University, Ajman, United Arab Emirates; and Departments of ${ }^{2}$ Ear, Nose, and Throat, ${ }^{3}$ Pathology, and ${ }^{4}$ Neuroscience, American Hospital \\ Dubai, Dubai, United Arab Emirates
}

\begin{abstract}
BACKGROUND Osteomas of the paranasal sinuses are benign, slow-growing bone tumors that can cause a variety of clinical features depending on their size and location. Most osteomas are asymptomatic and located in the frontal sinus. In rare cases, they may grow to extend into the cranial or orbital cavities, resulting in atypical presentations. The authors presented an aggressive case of a frontoethmoidal sinus osteoma with intracranial extension of an inflammatory sinonasal polyp.

OBSERVATIONS A 30-year-old man with a history of chronic sinusitis presented to the hospital after three episodes of loss of consciousness, chronic worsening of headache, and decreased sense of smell. Rhinoscopic examination showed mucosal polyps arising from the infundibulum and the superior meatus. Computed tomography showed a fibro-osseous mass in the left frontal sinus. Subsequent brain magnetic resonance imaging with and without contrast revealed a large, septated intracranial left frontal lesion approximately $6.5 \mathrm{~cm}$ in diameter that was compressing the underlying brain parenchyma.
\end{abstract}

LESSONS Intracranial extension of frontal sinus osteomas can have dire neurological implications. Early detection of lesions obstructing the paranasal sinuses outlet could prevent intracranial extension of the disease. The surgical approach to such tumors may be endonasal, open cranial, or a combination of both.

https://thejns.org/doi/abs/10.3171/CASE21105

KEYWORDS osteoma; frontal sinus; polyp; seizures

Osteomas are benign, slow-growing bone tumors most commonly seen in bones of the skull or in the mandible. They are also the most common benign neoplasms of the paranasal sinuses, with a prevalence of $3.69 \%$. $^{1,2}$ Most osteomas are located in the frontal sinus. ${ }^{3,4}$ Various theories (e.g., developmental, traumatic, infective) exist to explain the pathogenesis of osteomas; however, the exact etiology remains controversial. ${ }^{2,4,5}$ Although osteomas are usually asymptomatic lesions that are managed conservatively, complete excision is the treatment of choice for symptomatic paranasal sinus osteomas with orbital or intracranial extension. ${ }^{6}$ Surgery can be performed by an endonasal, open cranial, or combined approach, depending on the size and location of the tumor. Reconstruction of the anterior skull base is occasionally needed after resection.

We present here a rare and aggressive case of a frontoethmoidal sinus osteoma with intracranial extension of an inflammatory sinonasal polyp in a 30-year-old man who presented with neurological symptoms. The patient successfully underwent combined cranial and endonasal surgery for treatment of the mass and subsequent sinus pathway reconstruction.

\section{Illustrative Case}

A 30-year-old man presented to the hospital after three episodes of loss of consciousness, chronic worsening of frontal headache, and decreased sense of smell through the left nostril. He had a history of chronic sinusitis with left-sided nasal congestion. Rhinoscopic examination showed diffuse congested mucosa and mucosal polyps arising from the infundibulum and the superior meatus. No pathological or watery secretion was noted or reported.

Computed tomography (CT) of the paranasal sinuses and skull base (Fig. 1) showed a fibro-osseous mass in the left frontal sinus

ABBREVIATIONS CT = computed tomography; EEG = electroencephalogram; $\mathrm{MRI}$ = magnetic resonance imaging INCLUDE WHEN CITING Published May 24, 2021; DOI: 10.3171/CASE21105.

SUBMITTED February 16, 2021. ACCEPTED February 24, 2021.

(c) 2021 The authors, CC BY-NC-ND 4.0 (http://creativecommons.org/licenses/by-nc-nd/4.0/). 
with interruptions of the left ethmoid roof and slight thickening of the left frontal calvarial bone. The posterior frontal sinus wall appeared only partially present. Magnetic resonance imaging (MRI) of the brain with and without contrast (Fig. 2) was performed. It showed a large, septated intracranial left frontal lesion approximately $6.5 \mathrm{~cm}$ in diameter. It had heterogeneous signal and was mainly hyperintense on T2-weighted imaging and hypointense on T1-weighted imaging and fluid-attenuated inversion recovery. It contained bone with a multilobular enhancing configuration compressing the underlying brain parenchyma in the left frontal lobe and left frontal horn with no evidence of diffusion restriction. Coronal and sagittal image reconstructions indicated suspicion for a possible anterior cranial base defect. An electroencephalogram (EEG) showed arrhythmic 2- to 3-Hz delta activity in the left frontal area. Levetiracetam was started for treatment of suspected seizure activity.

A combined cranial and endonasal surgical approach was chosen to treat the lesion. A lumbar drain was placed, and a bicoronal incision was made to harvest a large, pedicled pericranial flap. A bifrontal craniotomy, accentuated on the left side, was performed. The basal frontal osteotomy was kept as low as possible to the lesion. A reciprocating saw was used over the frontal bone covering the osteoma to break the outer table. Once the bone flap was lifted, a polypoid translucent lesion that extended intradurally was evident. It had a distinct plane with the brain parenchyma and was completely resected. The large bony lesion on the frontal sinus was also exposed and resected en bloc. The bone of the left anterior cranial fossa and posterior wall of the frontal sinus had sclerotic consistency, and remodeling in response to the expanding mass was appreciated. Nevertheless,

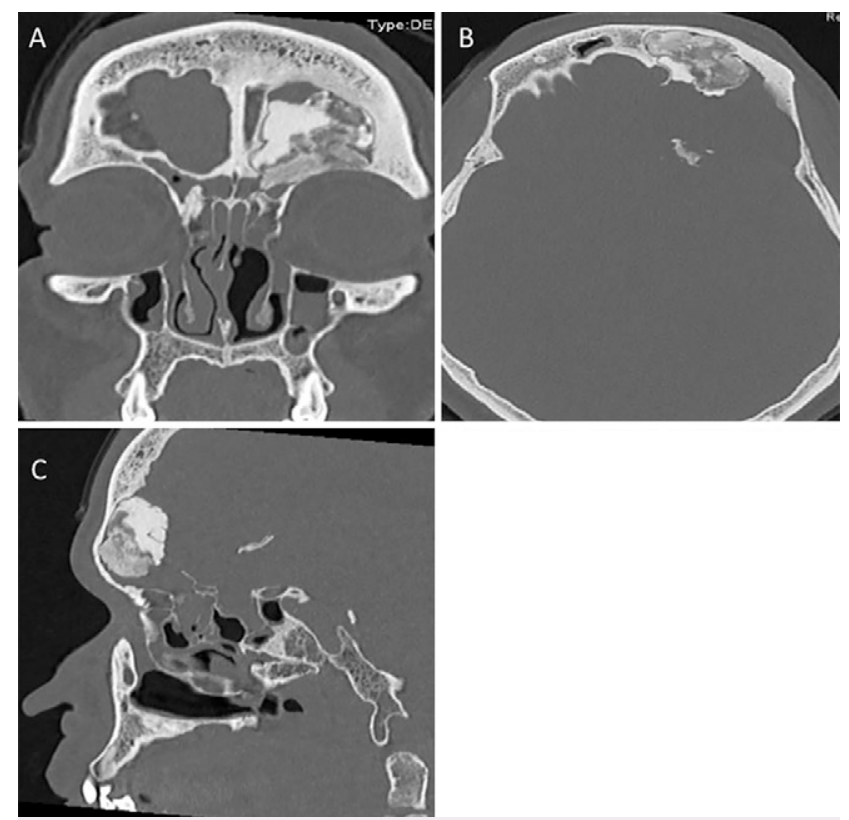

FIG. 1. CT scans of the paranasal sinuses, bone window. A: The skull base shows a fibro-osseous mass in the left frontal sinus with interruptions of the left ethmoid roof and slight thickening of the left frontal calvarial bone. $\mathbf{B}$ : In the axial cut, the posterior wall of the frontal sinus is pushed back and there is remodeling of the lateral frontal bone. C: Sagittal view shows remodeling and opacification of the ethmoid cells. no obvious breach into the nasal cavity was seen. The frontal sinus was cranialized, and the anterior skull base was reconstructed with the pedicled pericranial flap that was fixed to the sphenoid bone using 3-mm mini screws. To complete the procedure, we performed a bilateral endoscopic anteroposterior ethmoidotomy with medial antrostomy and left sphenoidotomy. No obvious connection with the intracranial compartment was found.

The excised intracranial lesion was polypoid tan with glistening mucosal lining (Fig. 3). The histopathology report indicated that the section of the intracranial polypoid decalcified lesion had respiratory mucosa lining (Fig. 4). The lesion in the frontal sinus was consistent with osteoma.

The patient recovered well and had an uneventful postoperative course. At the 6-week follow-up, he reported marked improvement of his nasal congestion. His headaches have resolved, his sense of smell has returned, and he has not experienced any seizures. CT of the paranasal sinuses and MRI of the brain (Fig. 5) show good reconstruction of the cranial base with complete resolution of the area of brain compression and only mild underlying encephalomalacia. Six months after the surgery, an EEG showed the presence of intermittent spike and wave epileptiform discharges in the left frontotemporal area with occasional spreading. Therefore, antiepileptic treatment was continued.

\section{Discussion}

Osteomas of the paranasal sinuses are benign, slow-growing bone tumors that can cause a variety of clinical features depending on their size and location. Most are asymptomatic and are discovered incidentally on imaging studies. Nevertheless, osteomas of the paranasal
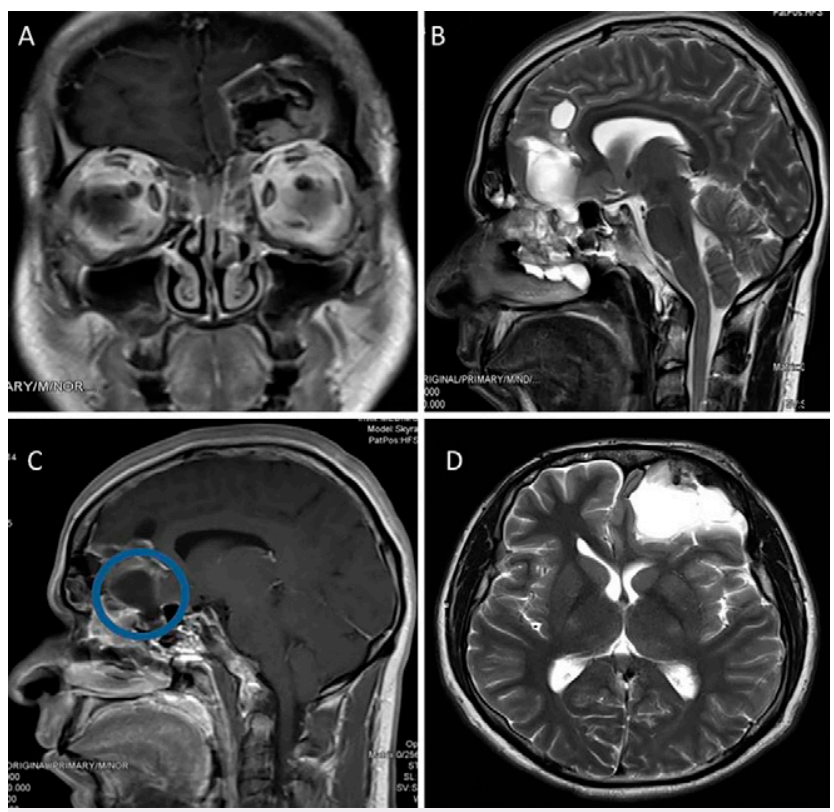

FIG. 2. Brain MRI with and without contrast shows a large intracranial septated left frontal lesion of approximately $6.5 \mathrm{~cm}$ in diameter (A, B, and $\mathrm{D}$ ). There is mainly hyperintense heterogeneous $\mathrm{T} 2$ signal and hypointense $\mathrm{T} 1$ signal, showing a bony lesion with multilobular enhancing configuration compressing the underlying brain parenchyma. In the blue circle (C), the suspected breach of the anterior fossa is shown. 


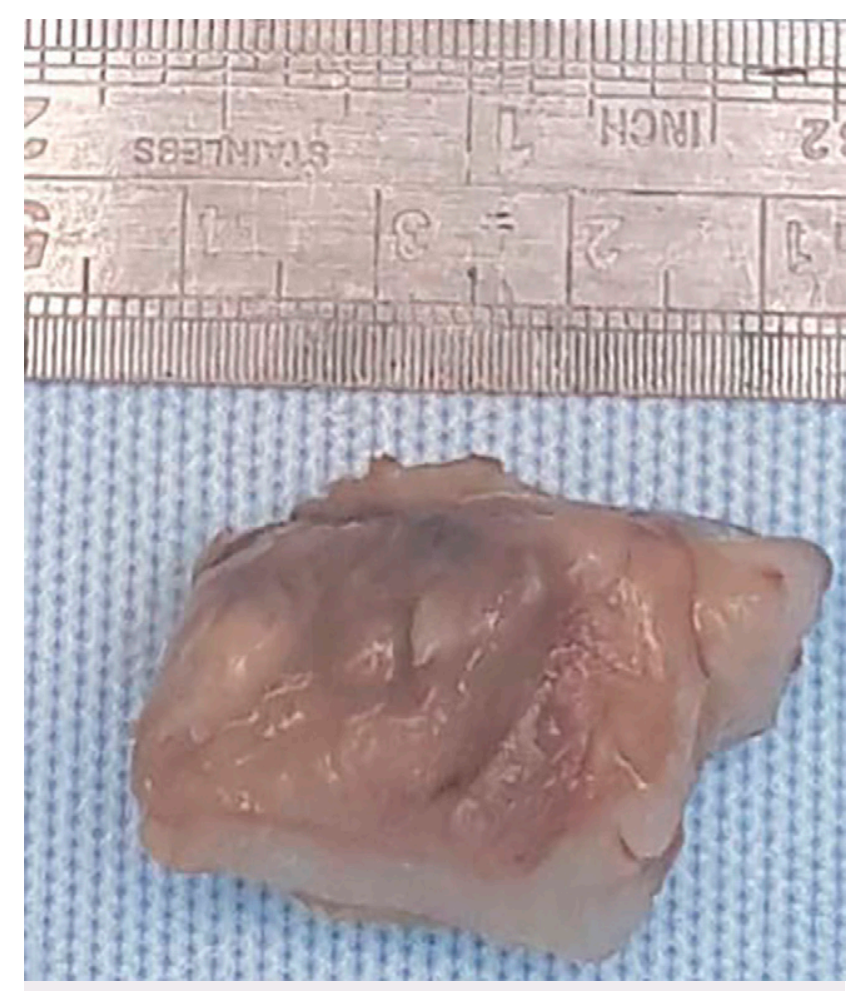

FIG. 3. The lesion is polypoid tan with glistening mucosal lining. The stroma showed presence of calcium/bone and required decalcification before sectioning of the tissue for histological examination.
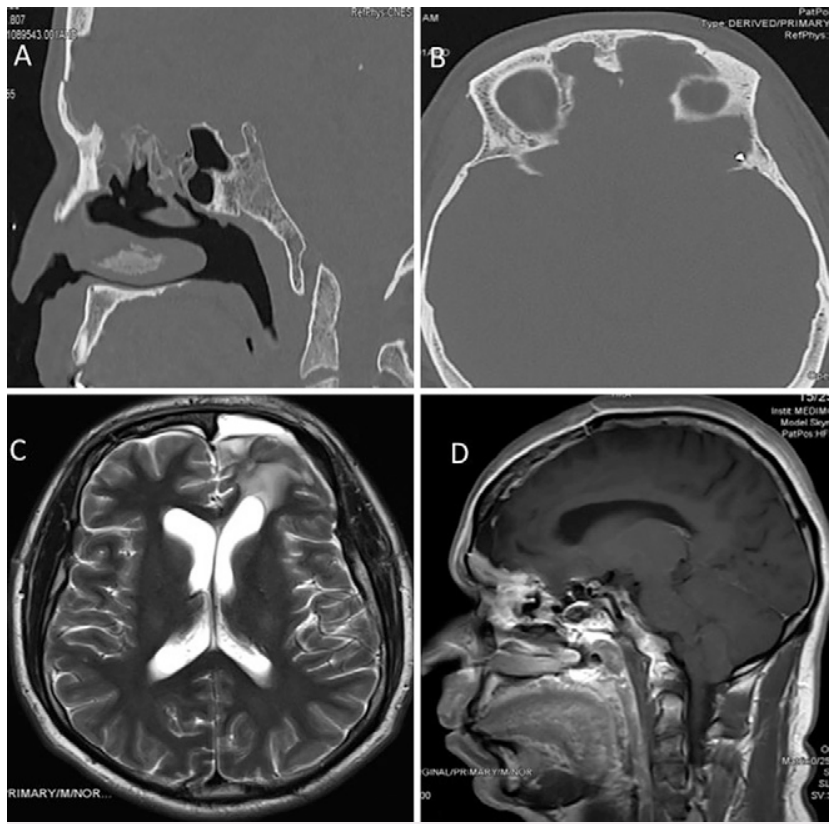

FIG. 5. Postoperative CT (A and B) shows adequate cranialization of the frontal sinus and improved pneumatization of the ethmoid sinus. Postoperative MRI (C and $\mathbf{D}$ ) shows reexpansion of the left frontal lobe and resolution of the midline shift (D). The pericranial flap can be noted as an enhancing lining that extends to the posterior ethmoid cells. sinuses may become symptomatic by direct mass effect or by obstructing normal sinus drainage. Only approximately $4 \%$ to $10 \%$ of all osteomas produce clinical symptoms, with osteomas of the frontoethmoidal region associated with earlier symptoms. ${ }^{4}$ The most common presenting symptoms include frontal sinusitis and headaches. ${ }^{3-5}$ Osteoma is also often associated with nasal polyposis and mucocele, which are considered secondary lesions. ${ }^{5}$ Posterior growth of frontal sinus osteomas may lead to intracranial involvement, and in the case of dural

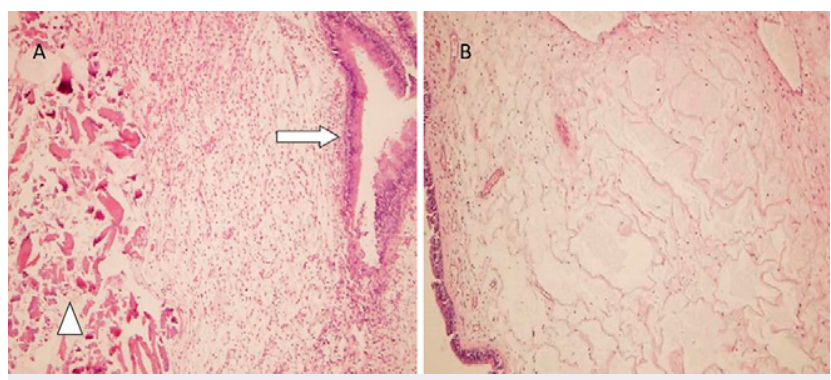

FIG. 4. A: Original magnification $\times 20$. Hematoxylin and eosin stain demonstrates a section of the intracranial polypoid decalcified lesion with respiratory mucosa lining (arrow). The stroma is focally edematous with areas of calcified tissue and osteoid metaplasia (arrowhead). B: Original magnification $\times 10$. Hematoxylin and eosin stain of nasal portion of the lesion shows typically myxoid edematous stroma with respiratory lining seen in inflammatory sinonasal-type polyps. penetration, complications such as cerebrospinal fluid fistula, meningitis, pneumocephalus, or frontal abscess can occur. ${ }^{6-8}$

\section{Observations}

In our case, the patient presented with generalized seizures. However, he did have symptoms of sinusitis for a long period of time before his diagnosis. The etiology of osteomas is not established, and several theories (e.g., traumatic, infectious, genetic, and developmental) have been put forth to explain their occurrence. According to the infectious theory, chronic inflammation triggers bone formation by stimulating proliferation of osteoblasts within the sinus mucoperiosteum. Association of chronic inflammatory conditions such as nasal polyposis and chronic rhinosinusitis with osteoma development has been described. Nevertheless, it is not clear whether the chronic inflammation or osteoma formation is the initiating event. $^{5,6}$

Grossly, osteomas are round or oval, hard, tan-white, and well circumscribed and are attached to the underlying bone by a broad base or occasionally by a small stalk. ${ }^{9}$ They are best diagnosed by $\mathrm{CT}$, which shows a rounded, well-circumscribed homogeneous radiodensity. In cases in which intracranial involvement is suspected, MRI is recommended. ${ }^{6}$

\section{Lessons}

Controversy surrounds the need for surgical intervention in asymptomatic osteomas; however, in symptomatic cases or in the case of orbital or intracranial involvement, surgical treatment is obligatory. The 
goal is to achieve complete en bloc resection, protect vital structures, allow adequate drainage of obstructed sinus contents, and optimize aesthetic outcome. ${ }^{10}$ An endonasal, external, or combined surgical approach can be used, depending on the location and size of the tumor. The endonasal approach is usually not indicated for giant osteomas and those located far lateral in the frontal sinus or widely attached to the skull base. ${ }^{11}$ Also, the eburnean consistency of many of these lesions might make it difficult to achieve complete resection via an endonasal approach. The external approach, whether by bicoronal scalp flap surgery, frontal craniotomy, or sometimes a lateral rhinotomy, is preferred for giant osteomas. ${ }^{8}$ In this case of a giant osteoma with intracranial extension, reconstruction of the anterior skull base would also be required, making the external approach more preferable. Despite adequate treatment of our patient's condition, the EEG still showed persistent epileptiform activity, which might render him on lifelong antiepileptic medication. Early detection of the osteoma obstructing the frontal sinus outlet may have prevented the intracranial extension of his disease.

\section{References}

1. Janovic A, Antic S, Rakocevic Z, Djuric M. Paranasal sinus osteoma: is there any association with anatomical variations? Rhinology. 2013; 51(1):54-60.

2. Georgalas C, Goudakos J, Fokkens WJ. Osteoma of the skull base and sinuses. Otolaryngol Clin North Am. 2011;44(4):875-890.

3. Mali S. Paranasal sinus osteoma: review of literature. Oral Surg. 2013;7(1):3-11.

4. Umur AS, Gunhan K, Songu M, et al. Frontal sinus osteoma complicated with intracranial inflammatory polyp: a case report and review of the literature. Rev Laryngol Otol Rhinol (Bord). 2008;129(4-5): 333-336.

5. Girolamo SD, Flora B, Passali FM, et al. Is there any association between nasal polyposis and osteoma? A retrospective analysis of incidence of paranasal sinus osteoma among 600 patients treated for nasal polyposis. Arch Otolaryngol Rhinol. 2019;5(2):35-39.

6. Stucker F, de Souza C, Kenyon GS, et al. Rhinology and Facial Plastic Surgery. Springer-Verlag; 2009.

7. Licci M, Zweifel C, Hench J, et al. Frontoethmoidal osteoma with secondary intradural mucocele extension causing frontal lobe syndrome and pneumocephalus: case report and review of literature. World Neurosurg. 2018;115:301-308.

8. Cheng KJ, Wang SQ, Lin L. Giant osteomas of the ethmoid and frontal sinuses: clinical characteristics and review of the literature. Oncol Lett. 2013;5(5):1724-1730.

9. Nielsen GP, Rosenberg AE. Update on bone forming tumors of the head and neck. Head Neck Pathol. 2007;1(1):87-93.

10. Romano A, Dell'Aversana OG, Pansini A, et al. Endoscopic approach for paranasal sinuses osteomas: our experience and review of literature. Oral Maxillofac Surg Cases. 2019;5(2):100094.

11. Ohta N, Suzuki $Y$, Wakli $T$, et al. Exploring the limits of the endoscopic approach to frontal sinus osteoma. Otolaryngology. 2013;3:140.

\section{Disclosures}

The authors report no conflict of interest concerning the materials or methods used in this study or the findings specified in this paper.

\section{Author Contributions}

Conception and design: Navarro, Poletti, Han. Acquisition of data: Navarro, Poletti, Han. Analysis and interpretation of data: Navarro, Poletti, Han. Drafting the article: all authors. Critically revising the article: all authors. Reviewed submitted version of manuscript: Navarro Farah, Han. Approved the final version of the manuscript on behalf of all authors: Navarro. Administrative/technical/material support: Navarro. Study supervision: Navarro.

\section{Correspondence}

Ramon Navarro: American Hospital, Dubai, United Arab Emirates. navarro.balbuena@gmail.com. 